\title{
Cerebral Glucose Metabolism after Portacaval Shunting in the Rat Patterns of Metabolism and Implications for the Pathogenesis of Hepatic Encephalopathy
}

\author{
Alan H. Lockwood,* Myron D. Ginsberg,‡ Howard M. Rhoades, $\$$ and Maria T. Gutierrezł \\ Departments of Neurology,* Psychiatry and Behavioral Sciences, $\S$ and Positron Diagnostic and Research Center,* \\ University of Texas Health Science Center at Houston, Houston, Texas 77225; and Department of Neurology, $\ddagger$ \\ University of Miami School of Medicine, Miami, Florida 33101
}

\begin{abstract}
The regional cerebral metabolic rate for glucose was measured in normal and portacaval shunted rats and the effects of unilateral carotid infusions of "threshold" amounts of ammonia were assessed. 8 wk after shunting the glucose metabolic rate was increased in all 20 brain regions sampled. Effects on subcortical and phylogenetically older regions of the brain were most pronounced with a $74 \%$ increase observed in the reticular formation at the collicular level. Increases in the cerebral cortex ranged from 12 to $18 \%$. Unilateral infusions of ammonia did not affect behavior but altered the electroencephalogram and selectively increased the glucose metabolic rate in the thalamus, hypothalamus, and substantia nigra in half of the animals, a pattern similar to that seen after a portacaval shunt, suggesting hyperammonemia as the cause of postshunt increases in glucose metabolism. Visual inspection of autoradiograms, computed correlation coefficients relating interregional metabolism, and principal component analysis suggest that normal cerebral metabolic and functional interrelationships are altered by shunting. Ammonia stimulation of the hypothalamic satiety centers may suppress appetite and lead to cachexia. Reductions in the ammonia detoxification capacity of skeletal muscle may increase the probability of developing future episodes of hyperammonemia, perpetuating the process. Direct effects of ammonia on specific brain centers such as the dorsomedial hypothalamus and reticular activating system may combine with global disruptions of cerebral metabolic-functional relationships to produce the protean manifestations of portal-systemic encephalopathy.
\end{abstract}

\section{Introduction}

Hyperammonemia is strongly implicated in the pathogenesis of hepatic or portal-systemic encephalopathy (1-8). In humans, this disorder is characterized by an altered level of consciousness, impaired cognitive functions, occasional movement disorders, abnormal muscle tone, hyperactive deep tendon reflexes, and an extensor plantar sign, due to corticospinal tract dysfunction. Hyperammonemia develops as ammonia-rich hepatic portal blood enters the systemic circulation via shunts, without passing through the liver where it is normally detoxified. Ammonia dif-

Portions of this work were presented at the 107th annual meeting of the American Neurological Association, 30 September to 2 October, 1982. Address reprint requests and correspondence to Dr. Lockwood.

Received for publication 16 September 1985 and in revised form 2 March 1986.

J. Clin. Invest.

(C) The American Society for Clinical Investigation, Inc.

$0021-9738 / 86 / 07 / 0086 / 10 \$ 1.00$

Volume 78, July 1986, 86-95 fuses across the blood-brain barrier with blood-brain $\mathrm{pH}$ gradients (9) and regional variations in the permeability-surface area products of the barrier to ammonia (10) modulating ammonia uptake. Hyperammonemia produces a variety of neurochemical and physiological abnormalities including elevation of brain ammonia and glutamine levels, decreases in glutamate (3), and a reduction in ATP levels in the reticular formation at the level of the inferior colliculus (5). Neuropathological changes are confined to astrocytes, giving them characteristics of metabolically hyperactive cells (6). Chronic shunt-induced hyperammonemia leads to the development of hypersensitivity to exogenous ammonia, as suggested by the clinical observations of Walker and Schenker (7) and blood flow and metabolic data from rats with portacaval shunts reported by Gjedde et al. (2).

Because of the central importance of glucose metabolism in cerebral energy production, strong links between regional cerebral glucose metabolism and brain function, and conflicts in the literature concerning the effects of ammonia and portacaval shunting on cerebral metabolism, we measured the cerebral glucose metabolic response to portacaval shunt construction in the rat, evaluated the effect of selective "threshold" infusions of ammonia, administered via the carotid artery, and assessed the effect of portacaval shunts on metabolic interrelationships within the brain-its "metabolic anatomy." We confirmed the observations of Cruz and Duffy (8), who reported that most cerebral structures increased their rate of glucose metabolism 8 wk after shunting. We observed the largest increase in the reticular formation at the level of the colliculi, the portion of the brain implicated in the maintenance of consciousness. Although there were no discernible gross behavioral effects of threshold intracarotid infusions of ammonia, the cerebral metabolic rate for glucose was increased in a pattern similar to the increases observed after portacaval shunting in nearly half of the infused animals, suggesting that hyperammonemia is responsible for the increases observed after shunting. Finally, we used multivariate statistical techniques to evaluate metabolic interrelationships in the brain and concluded that shunting alters fundamental patterns of cerebral metabolism and presumably functional interrelationships. A preliminary report of these studies has been published (11).

\section{Methods}

Animal preparation. Male Wistar rats, 250-300 g, were used. End-toside portacaval shunts were constructed by the method of Lee and Fisher (12). $8 \mathrm{wk}$ postoperatively, and after an overnight fast, the animals were anesthetized with ether, paralyzed with curare, and ventilated via a tracheostomy with $70 \% \mathrm{~N}_{2} \mathrm{O}$ and $30 \% \mathrm{O}_{2}$ so that normal blood gas values could be maintained in all animals and possible effects of ammonia on ventilatory control could be avoided. Catheters were placed in the femoral artery and vein. Arterial blood was sampled and the ventilator adjusted to normalize blood gas values. In infused animals, a polyethylene (PE-10) catheter was placed in the external carotid artery and advanced 
to the carotid bifurcation. A ligature was placed around the catheter at the bifurcation to direct all infused solutions into the common carotid artery. Care was taken to avoid trauma to the carotid sinus, the carotid body, their nerves, and blood supplies. Saline, $0.9 \%$, or ammonium acetate, $0.2 \mathrm{M}$, was infused via the catheter at $0.007 \mathrm{ml}$ per minute with a Harvard pump (Harvard Apparatus Co., Inc., S. Natick, MA) and a 5 $\mathrm{ml}$ glass syringe. In half of the animals the electroencephalogram was monitored during the infusions. Arterial blood ammonia levels were measured by the method of Folbergrova et al. (13).

Two normal and two portacaval shunt animals were fitted with external carotid artery catheters under halothane general anesthesia. After a 4-h recovery period, an ammonia infusion was started as described above. These alert, unrestrained animals were observed for the development of behavioral abnormalities (circling, seizures, coma, etc.) for $1 \mathrm{~h}$. Gentle stimuli were applied to prevent sleep during the observation period.

To quantify the ammonia load imposed by the infusions, four additional normal animals were paralyzed, ventilated, and prepared with external carotid artery catheters, as described above. These animals were infused with cyclotron-produced ${ }^{13} \mathrm{~N}$-labeled $0.2 \mathrm{M}$ ammonium acetate (the specific activity of this solution changed rapidly due to the 10-min half-life of ${ }^{13} \mathrm{~N}$ while the ammonium acetate concentration remained $0.2 \mathrm{M})$. Details of the ${ }^{13} \mathrm{~N}$-ammonia preparation have been published (9). After a 10-min infusion, the animals were killed and the infused hemisphere was split into four portions in the coronal plane and isotope content was measured in a well counter. Appropriate corrections were made for isotope half-life.

Glucose metabolic rate measurement. The method of Sokoloff et al. (14) was used. Briefly, an i.v. bolus of $100 \mu \mathrm{Ci} / \mathrm{kg}$ of $2-\left[2-{ }^{14} \mathrm{C}\right]-$ deoxy-Dglucose (DG), ${ }^{1}$ was injected into anesthetized, paralyzed, ventilated animals after sequential blood gas measurements confirmed steady state conditions. Intracarotid infusions were started $15 \mathrm{~min}$ before the DG injection. $\sim 30$ arterial blood samples were obtained during the next 45 min, with at least six samples collected in the first minute. The plasma DG content was measured by liquid scintillation spectrometry using an external standard to correct for quenching. The plasma glucose was measured at four equally timed intervals with a Glucose Analyzer II (Beckman Instruments, Inc., Fullerton, CA). The animals were killed 45 min after the injection of the DG. The brains were removed rapidly and frozen in Freon 22 , cooled to $-70^{\circ} \mathrm{C}$ with liquid nitrogen. Serial sections $20 \mu \mathrm{m}$ thick were cut in a cryostat maintained at $-20^{\circ} \mathrm{C}$. The tissue sections were mounted on coverslips, dried, and placed next to the emulsion side of Kodak SB-5 x-ray film (Eastman Kodak Co., Rochester, NY) along with a set of calibrated ${ }^{14} \mathrm{C}$-methyl-methacrylate standards. When possible, adjacent tissue sections were stained with thionin. The optical density of the developed autoradiograms was measured with a rotating drum digitizer (Optronics International, Inc., Chelmsford, MA). The regional brain ${ }^{14} \mathrm{C}$ content was calculated using the standards, and the regional glucose metabolic rate was computed for each point on the autoradiogram using the equation of Savaki et al. (15) with the rate constants and the lumped constant values published by Sokoloff et al. (14). Regional glucose metabolic rate images were displayed with a Grinnel image-processing system using a pseudo-color scheme similar to that described by Goochee et al. (16). Regions of interest on the metabolic images were identified with the aid of the thionin-stained sections and an atlas of rat brain anatomy (17). Glucose metabolic rates were extracted and tabulated.

Statistical analyses. Differences in the glucose metabolic rate in specific brain nuclei from the infused vs. the noninfused side of the brain were sought using the paired $t$ test. Preinfusion vs. postinfusion arterial ammonia concentrations were also compared with this test. Differences between mean metabolic rates observed in normal and portacaval shunt animals were determined with Student's $t$ test.

Additional analyses of metabolic relationships were conducted using normalized regional metabolic values. Normalization was accomplished

1. Abbreviations used in this paper: DG, $2-\left[2-{ }^{14} \mathrm{C}\right]-$ deoxy-D-glucose; $r_{\mathrm{c}}$, congruence coefficient. by dividing the measured metabolic rate in each brain region of a given animal by the mean metabolic rate for all structures sampled in that animal. This eliminates variations that are attributable to the fact that some animals had metabolic rates that were uniformly high or low for all brain regions but has no effect on comparisons of the metabolic rate from one region in the animal to other regions for the same animal.

A principal component analysis among 20 regions in normal animals and 19 regions in portacaval shunt animals was conducted using the individual normalized values as described by Overall and Klett (18). Two factors, arbitrarily labeled A and B, were extracted from both the normal and portacaval shunt groups. The congruence coefficient, $r_{\mathrm{c}}$, was computed to determine whether the extracted factors A and B in the normal animals were similar or different from the factors extracted from the portacaval shunt animals using procedures described by Cattel (19). The brachium conjunctivum for portacaval animals was excluded from this analysis because of the relatively small size of that sample $(n=4)$.

The normalized values were used to compute a 20 by 20 correlation matrix to display the correlation between the metabolic rate in one region with the rate in all other sampled regions. For the sake of simplicity, only those correlation coefficients with $P$ values less than or equal to 0.05 are included. A test for differences between individual correlation coefficients from the normal animals vs. the portacaval shunt animals was conducted as described by Bruning and Kintz (20).

\section{Results}

\section{Physiological effects of ammonia infusion}

A total of 14 normal and 9 portacaval shunt animals were studied with the DG method. Arterial ammonia levels in eight normal animals averaged $0.088 \pm 62 \mathrm{mM}(\mathrm{SD})$ and $0.296 \pm 0.122 \mathrm{mM}$ in four portacaval shunt animals. Paired $t$ tests showed that intracarotid ammonia and saline infusions did not alter the systemic arterial ammonia concentration. Physiological data are shown in Table I.

Alert, unrestrained, normal and portacaval shunt animals did not exhibit any behavioral abnormalities before or during the 1-h infusion of ammonium acetate at the specified rate. Large increases in the infusion rate produced facial twitching followed by generalized seizures in all animals, confirming proper catheter placement. About half of the animals that were paralyzed, ventilated, and infused with ammonia, developed slowing of the electroencephalogram that was observable over the infused hemisphere.

We based our ammonia infusion schedule on our desire to dissect any primary metabolic effects of ammonia on glucose metabolism from secondary effects due to epileptic seizures or

Table 1. Physiological Data

\begin{tabular}{|c|c|c|c|c|}
\hline & \multicolumn{2}{|l|}{ Control } & \multicolumn{2}{|c|}{ Portacaval shunt } \\
\hline & Preinfusion & Postinfusion & Preinfusion & Postinfusion \\
\hline \multicolumn{5}{|l|}{ Plasma glucose } \\
\hline$(m M)$ & $10.4 \pm 2.1$ & - & $9.0 \pm 2.7$ & - \\
\hline \multicolumn{5}{|l|}{ Arterial blood } \\
\hline pH & $7.42 \pm 0.06$ & - & $7.44 \pm 0.12$ & - \\
\hline $\mathrm{PCO}_{2}$ & $36.9 \pm 3.7$ & - & $36.0 \pm 3.0$ & - \\
\hline $\mathrm{PO}_{2}$ & $124 \pm 19$ & - & $138 \pm 24$ & - \\
\hline \multicolumn{5}{|l|}{ Blood ammonia } \\
\hline ( $\mu \mathrm{mol} /$ /liter) & $88 \pm 62$ & $89 \pm 63^{*}$ & $278 \pm 144$ & $305 \pm 109^{*}$ \\
\hline
\end{tabular}

All values are means \pm SD.

* Differences not significantly different from preinfusion values. 
coma. Assuming that the normal animals had a cerebral blood flow of $0.9 \mathrm{ml} \cdot \mathrm{g}^{-1} \cdot \mathrm{min}^{-1}(2)$ and a cerebral ammonia extraction of $45 \%$ (9), the expected ammonia uptake and metabolism rate would be $0.035 \mu \mathrm{mol} \cdot \mathrm{g}^{-1} \cdot \mathrm{min}^{-1}$. From the results of 12 brain samples obtained from three animals infused with ${ }^{13} \mathrm{~N}$-ammonia of known specific activity, we calculated that an additional $0.054 \pm 0.038 \mu \mathrm{mol} \cdot \mathrm{g}^{-1} \cdot \mathrm{min}^{-1}$ of ammonia was taken up and metabolized by the cerebral hemisphere on the infused side.

\section{Studies of cerebral glucose metabolism}

Definition of experimental groups. Paired $t$ tests were used to compare glucose metabolic rates in the saline-infused vs. the noninfused hemisphere in normal and portacaval shunt animals: no interhemispheric differences were found. The saline infusion was thus without effect on cerebral glucose metabolism. The $t$ test for differences between means was used to compare metabolic rates for structures in the noninfused hemisphere of normal animals infused contralaterally with saline with the same structure in the noninfused hemisphere of normal animals given contralateral ammonium acetate. No differences were found for structures listed in Table II. A similar evaluation was made of metabolic rates in noninfused hemispheres of portacaval shunt animals receiving contralateral saline infusions in comparison with portacaval shunt animals receiving contralateral ammonium acetate infusions. Again, no significant differences were found. Therefore, since infusions of ammonia and saline did not affect glucose metabolism in the noninfused hemisphere, we pooled metabolic rate data from noninfused hemispheres and used them to evaluate the effects of the portacaval shunt on cerebral glucose metabolism. The resulting data are compiled in Table II.

\section{Effects of a portacaval shunt on regional cerebral glucose consumption}

Single region effects. The glucose metabolic rate was measured in 20 brain regions of noninfused hemispheres of normal and portacaval shunted animals. These regions included three areas of the cerebral cortex, 16 subcortical nuclei, and the brachium conjunctivum. The results, before and after normalization, are shown in Table II. The results are in general similar to those reported by Cruz and Duffy (8) who reported systematically higher metabolic rates in their unanesthetized animals restrained by plaster casts. For each site, the regional glucose metabolic rate was significantly higher in the portacaval shunt group, when compared with the rate measured in normal animals $(P<0.01)$. Regions of depressed glucose use were not observed. Dorsal, dorso-lateral, and lateral regions of the cerebral cortex, sampled in sections that included thalamus, showed the smallest increases in glucose metabolism, exceeding control rates by 18,12 , and $17 \%$, respectively. The greatest effect was measured in the brainstem reticular formation at the level of the inferior colliculus, where a $74 \%$ increase was observed. In general, the pattern of increased metabolism was very similar to that observed after the infusion of ammonium acetate. The most notable difference was an absence of a dramatic stimulation of metabolism in the substantia nigra such as that shown in Fig. 1. Nigral glucose metabolism was increased by $48 \%$ after a portacaval shunt.

Changes in metabolic patterns. Visual inspection of the metabolic rate images, as represented by the autoradiograms shown in portions $E$ through $H$ of Figs. 1 and 2 for normal and portacaval animals, suggested that the pattern of glucose metabolism was altered by the shunt. Evidence for pattern dissimilarity be-

Table II. Glucose Metabolic Rates in Brain Regions of Normal and Portacaval Shunted Rats

\begin{tabular}{|c|c|c|c|c|c|}
\hline \multirow[b]{2}{*}{ Brain region } & \multicolumn{2}{|l|}{ Control } & \multicolumn{2}{|l|}{ Portacaval shunt } & \multirow[b]{2}{*}{ Percent increase } \\
\hline & Raw & Normalized & Raw & Normalized & \\
\hline & $\mu \mathrm{mol} \cdot 100 \mathrm{~g}^{-1}$ & $\mu \mathrm{mol} \cdot 100 \mathrm{~g}^{-1} \cdot \mathrm{min}^{-1}$ & $\mu \mathrm{mol} \cdot \mathrm{g}^{-1} \cdot \mathrm{min}^{-1}$ & & \\
\hline Cortex-dorsal & $61.1 \pm 18.3(13)$ & $1.21 \pm 0.08$ & $72.2 \pm 8.3$ & $1.00 \pm 0.08$ & 18 \\
\hline Cortex-lateral & $71.7 \pm 19.4(14)$ & $1.45 \pm 0.14$ & $80.5 \pm 10.6(9)$ & $1.11 \pm 0.11$ & 12 \\
\hline Cortex-dorsolateral & $57.8 \pm 17.2(14)$ & $1.15 \pm 0.06$ & $68.3 \pm 7.8$ & $0.95 \pm 0.07$ & 17 \\
\hline Medial septal nucleus & $39.4 \pm 19.4(10)$ & $0.76 \pm 0.24$ & $57.2 \pm 13.9(9)$ & $0.79 \pm 0.18$ & 46 \\
\hline Globus pallidus & $45.5 \pm 22.8(13)$ & $0.86 \pm 0.24$ & $66.7 \pm 15.0(9)$ & $0.92 \pm 0.19$ & 54 \\
\hline Lateral septal nucleus & $27.8 \pm 11.1(13)$ & $0.52 \pm 0.13$ & $42.8 \pm 21.7(9)$ & $0.58 \pm 0.25$ & 46 \\
\hline Anterior hypothalamus & $32.2 \pm 14.4(14)$ & $0.62 \pm 0.16$ & $40.5 \pm 15.0(7)$ & $0.56 \pm 0.18$ & 26 \\
\hline Thalamus dorsomedial & $61.7 \pm 3.4$ & $1.25 \pm 0.16$ & $98.5 \pm 17.7(8)$ & $1.35 \pm 0.22$ & 59 \\
\hline Thalamus vent (ant-med) & $65.6 \pm 22.8(8)$ & $1.13 \pm 0.29$ & $86.7 \pm 11.1(6)$ & $1.21 \pm 0.08$ & 34 \\
\hline Thalamus vent (dorsal) & $12.8 \pm 21.7(14)$ & $1.42 \pm 0.26$ & $98.9 \pm 21.7(9)$ & $1.37 \pm 0.26$ & 39 \\
\hline Ventromedial hypothal. & $31.1 \pm 12.2(13)$ & $0.60 \pm 0.12$ & $48.3 \pm 13.3(9)$ & $0.66 \pm 0.13$ & 55 \\
\hline Thal. retic-reuniens & $42.2 \pm 19.4(10)$ & $0.82 \pm 0.18$ & $62.2 \pm 23.3(8)$ & $0.87 \pm 0.30$ & 37 \\
\hline Mammillary body & $39.4 \pm 13.3(11)$ & $0.77 \pm 0.19$ & $54.4 \pm 18.9(6)$ & $0.74 \pm 0.19$ & 38 \\
\hline Reticular formation & $48.3 \pm 12.8(14)$ & $0.77 \pm 0.15$ & $83.9 \pm 13.9(9)$ & $1.16 \pm 0.12$ & 74 \\
\hline Substantia nigra & $39.4 \pm 11.1(14)$ & $0.81 \pm 0.13$ & $58.3 \pm 8.9$ & $0.80 \pm 0.06$ & 48 \\
\hline Brachium conjunctivum & $41.1 \pm 13.3(8)$ & $0.84 \pm 0.17$ & $67.8 \pm 15.0(4)$ & $1.01 \pm 0.14$ & 65 \\
\hline Nuc. mes trigem tract & $51.1 \pm 13.9$ & $1.05 \pm 0.22$ & $75.0 \pm 18.9(9)$ & $1.03 \pm 0.19$ & 47 \\
\hline Deep cerebellar nuclei & $59.4 \pm 22.2(13)$ & $1.20 \pm 0.25$ & $97.8 \pm 19.4(9)$ & $1.37 \pm 0.27$ & 64 \\
\hline Superior colliculus & $57.8 \pm 25.0(9)$ & $1.12 \pm 0.22$ & $91.7 \pm 17.8(6)$ & $1.25 \pm 0.18$ & 59 \\
\hline Caudate-putamen & $63.9 \pm 19.4(9)$ & $1.28 \pm 0.12$ & $90.5 \pm 13.3(9)$ & $1.26 \pm 0.18$ & 42 \\
\hline
\end{tabular}

All values are means \pm SD. Numbers in parentheses are the sample size for the group. All portacaval shunt values are significantly greater than control, $P<0.01$. 



Figure 1. Normal animals. Thionin-stained sections (A-D) and autoradiograms (E-H) are from four representative coronal planes from a single animal infused with saline via the carotid artery. In most cases histological sections were immediately adjacent to the autoradiographic section. The autoradiograms I-L are from an animal infused with ammonium acetate into the hemisphere on the right as specified in Methods. Note the differences in the pattern of glucose metabolism

tween the two groups can be seen in the results of the principal components analysis shown in Table III. This table lists the correlations between the normalized regional metabolic rates and the extracted, hypothetical components. Objective criteria are not available to describe region-by-region results of this analysis, however, inspection of the coefficients (magnitude and sign) and whether the first or second extracted factor dominates (greatest absolute magnitude) provides insight into the degree of similar- in $\mathrm{E}-\mathrm{H}$ in this figure compared with the same components of Fig. 2 after portacaval shunting. Abbreviations: $A q$, aqueduct of Sylvius; $C P u$, Caudate-putamen; $M D$, medial dorsal nucleus of thalamus; $M G$, medial geniculate nucleus; $R$, red nucleus; $S N R$, reticular portion of substantia nigra; $V M$, ventromedial nucleus of thalamus; $V M H$, ventromedial nucleus of hypothalamus; VPM/L, medial and lateral ventroposterior nucleus of thalamus.

ities or differences between the groups. The factor variance, shown at the bottom of each column, indicates the amount of covariance explained by the extracted hypothetical factor; the larger the factor variance, the more covariance accounted for by the extracted factor. The congruence coefficient, $r_{\mathrm{c}}$, was computed to compare the congruence or similarity of factors $A$ and $B$ from the normal animals with factors $A$ and $B$ from the portacaval animals yielding values of $0.445(0.05<P<0.10)$ and 

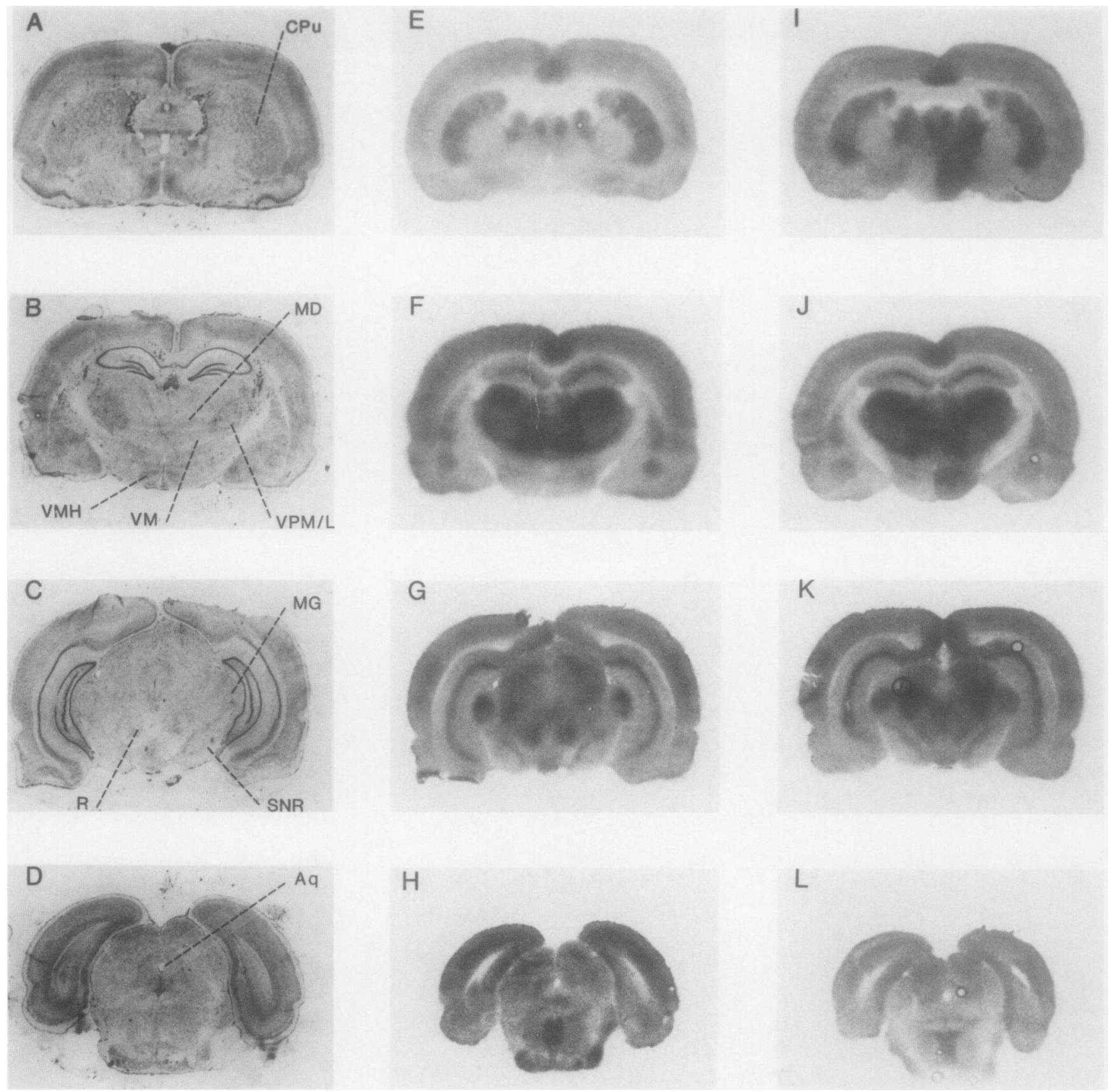

Figure 2. Portacaval shunt animals. Thionin-stained sections (A-D) and autoradiograms (E-H) are from four representative coronal planes from a single animal infused with saline via the carotid artery. In most cases histological sections were immediately adjacent to the autoradiographic section. The autoradiograms $\mathrm{I}-\mathrm{L}$ are from an animal infused with ammonium acetate into the hemisphere on the right as specified in Methods. The overall glucose metabolic rate increases after portaca-

$-0.233(P>0.10)$, respectively. Both of these $P$ values are greater than $\mathbf{0 . 0 5}$, and therefore congruence or similarity is not present. Since the test for congruence fails to reach statistical significance, we conclude that the groups are not similar.

Table IV presents individual correlation coefficients from the full set of 20 brain regions where the absolute value of $P$ was 0.05 or less and provides additional evidence for pattern dissimilarity between the two groups of animals. In the normal animals val shunting in a nonuniform fashion. Cerebral cortex was least affected with maximal effects observed in the reticular formation. Multivariate statistical testing suggests that the pattern of cerebral glucose use is altered from the normal state by a portacaval shunt. Note the stimulation of glucose metabolism seen in the ventromedial nucleus of the hypothalamus after ammonia infusion, an effect that may suppress appetite and cause cachexia. Abbreviations are as in Fig. 1.

there were 21 significant correlations of metabolic activity (12 positive and 9 negative), and 16 significant correlations in the portacaval shunt animals ( 8 positive and 8 negative). The patterns of significant correlations exhibited by the two groups do not appear to be very similar. For example, the mesencephalic nucleus of the trigeminal tract showed the greatest number of significant correlations (6) in the normal animals with positive correlations between two cortical regions and the deep cerebellar 
Table III. Principal Components Analysis

\begin{tabular}{|c|c|c|c|c|}
\hline \multirow[b]{2}{*}{ Brain region/factor } & \multicolumn{2}{|l|}{ Normal } & \multicolumn{2}{|c|}{ Portacaval shunt } \\
\hline & A & B & A & B \\
\hline Cortex, dorsal & 0.863 & -0.029 & 0.548 & -0.537 \\
\hline Cortex, lateral & 0.701 & 0.071 & 0.710 & -0.630 \\
\hline Cortex, dorso-lateral & 0.662 & 0.408 & 0.532 & -0.745 \\
\hline Medial septal nucleus & -0.802 & -0.461 & 0.327 & 0.679 \\
\hline Globus pallidus & -0.707 & -0.082 & 0.542 & -0.121 \\
\hline Lateral septal nucleus & -0.564 & -0.185 & -0.795 & 0.280 \\
\hline Hypothalamus, anterior & -0.682 & 0.193 & -0.315 & 0.472 \\
\hline Thalamus, dorsomedial & 0.147 & -0.453 & -0.197 & -0.031 \\
\hline $\begin{array}{l}\text { Thalamus, ventral N } \\
\text { (ant-med) }\end{array}$ & -0.656 & 0.717 & -0.718 & -0.779 \\
\hline $\begin{array}{l}\text { Thalamus, ventral } \mathrm{N} \\
\text { (dorsal) }\end{array}$ & 0.698 & -0.417 & 0.568 & 0.307 \\
\hline $\begin{array}{l}\text { Hypothalamus, } \\
\text { ventromedial }\end{array}$ & -0.682 & 0.335 & -0.811 & -0.166 \\
\hline $\begin{array}{l}\text { Thalamus, reticular- } \\
\text { reuniens }\end{array}$ & 0.345 & -0.492 & -0.525 & -0.556 \\
\hline Thalamus, mammillary & -0.401 & -0.243 & -0.728 & 0.472 \\
\hline Reticular formation & 0.235 & 0.599 & 0.687 & 0.454 \\
\hline Substantia nigra & 0.148 & 0.731 & 0.145 & -0.259 \\
\hline Brachium conjunctivum & 0.020 & -0.297 & $*$ & * \\
\hline $\begin{array}{l}N \text { mesenceph trigeminal } \\
\text { tract }\end{array}$ & 0.891 & -0.008 & -0.014 & -0.157 \\
\hline Deep cerebellar nuclei & 0.559 & 0.476 & 0.204 & -0.518 \\
\hline Superior colliculus & 0.002 & 0.694 & 0.548 & 0.478 \\
\hline Caudate-putamen & -0.532 & 0.184 & 0.639 & 0.192 \\
\hline Factor variance & 6.75 & 3.45 & 5.80 & 4.11 \\
\hline $\begin{array}{l}\text { Congruence coefficient, } \\
r_{\mathrm{c}} \text { normal vs. } \\
\text { portacaval }\end{array}$ & 0.445 & -0.233 & & \\
\hline Significance, $P$ & $>0.05$ & $>0.10$ & & \\
\hline
\end{tabular}

\section{* Not included in analysis.}

Principal components analysis for normal and portacaval shunt animals. The correlation coefficients between normalized regional metabolic rates and the extracted hypothetical factors $A$ and $B$ are shown without rotation. The congruence coefficient, $r_{c}$, tests for significant congruence between the coefficients $A$ and $B$, respectively, from the normal and portacaval animals. Since the $P$ values are both $>0.05$, the test for significant congruence fails, congruence is excluded, and we conclude that the groups are not similar.

nuclei and negative correlations with the globus pallidus, the ventromedial and anterior regions of the hypothalamus. This same region had no significant correlations in the portacaval shunt animals. In the portacaval shunt animal group the mammillary body showed five significant correlations: positive with the brachium conjunctivum and the anterior hypothalamus; and negative with the globus pallidus and two cortical regions. Normal animals exhibited no significant correlations with this nucleus.

Table $\mathrm{V}$ presents selected computed probability values associated with the test for differences between regional correlation coefficients. The first entry in the second column, 0.006 , indicates that the correlation between the lateral cortex and the globus pallidus in the normal group $(r=-0.568$, value shown in Table
IV) was significantly different from that in the portacaval shunt group ( $r=0.634$, value not shown in Table IV since this correlation did not reach the criterion for inclusion in the table) at a level of significance equal to 0.006 . There are 25 probability values of 0.050 or less out of a total of 180 probability values computed in the table. This represents approximately one regional interaction out of every eight, and provides additional evidence for differences between metabolic interactions in the normal vs. portacaval shunt animals.

\section{Effects of unilateral ammonium acetate infusions}

Since we chose to evaluate threshold effects of ammonia on cerebral metabolism, we were not surprised by the absence of a consistent, statistically significant effect of ammonium acetate on cerebral metabolism in the infused hemisphere (paired $t$ tests yielded $P$ values $>0.05$ ). We were unable to identify any variable associated with the exposure to ammonia, including blood ammonia concentration, plasma glucose concentration, blood pressure, and blood gas values. We believe that the variability of the ammonia infusion effect is attributable to the variations in the exogenous ammonia delivered to brain regions, as shown by the variance in the ${ }^{13} \mathrm{~N}$-ammonia infusion studies.

A distinct pattern of increased glucose use (with variations in intensity) was evident in those animals when visual inspection of the quantitative images of glucose metabolic rates indicated an asymmetry. Examples of thionin-stained histological sections and DG autoradiograms of normal animals receiving saline and ammonium acetate infusions are seen in Fig. 1, and of portacaval shunt animals similarly infused in Fig. 2. In anterior sections of normal animals infused with ammonium acetate, increases in glucose metabolism were seen in the anteromedial and medial nuclei of the thalamus. In more posterior sections, the ventral nucleus of the thalamus was activated along with hypothalamic nuclei. In sections containing the substantia nigra and the superior colliculus, activation was seen in these nuclei along with the red nucleus, the medial geniculate body, and posteriorly, in the reticular activating system around the aqueduct. The largest increases were observed in the substantia nigra. In the example shown in Fig. 1, the nigral metabolic rate was $197 \mu \mathrm{M} \cdot 100$ $\mathrm{g}^{-1} \cdot \mathrm{min}^{-1}$ in the infused side as compared with the mean control rate of $39 \pm 11 \mu \mathrm{M} \cdot 100 \mathrm{~g}^{-1} \cdot \min ^{-1}( \pm \mathrm{SD})$.

In portacaval shunt animals, the pattern of the additional increase in glucose metabolism associated with ammonium acetate infusion was similar to that observed in the normal animals, but less striking. The substantia nigra was less sensitive in this group in which maximal effects were produced in hypothalamic nuclei.

\section{Discussion}

Cerebral glucose metabolism during hyperammonemia or a portacaval shunt. Cirrhosis of the liver ranks sixth as a cause of lost years of life in the United States (21). During the terminal phases of the diseases that produce cirrhosis, repeated episodes of encephalopathy contribute to the morbidity and mortality of the condition. Convincing clinical and experimental evidence links hyperammonemia with the development of encephalopathy and coma (1-8). Ammonia-rich hepatic portal blood is shunted into the systemic circulation as a complication of portal hypertension, or as the result of surgical procedures. The resulting hyperammonemia contributes to the development of brain dysfunction. This study was designed to evaluate the changes in brain glucose 


\begin{tabular}{|c|c|c|c|c|c|c|c|c|c|c|c|c|c|c|c|c|c|}
\hline \multicolumn{18}{|c|}{ Normal animals } \\
\hline & 1 & 2 & 3 & 4 & 5 & 6 & 7 & 89 & 10 & 11 & 12 & 13 & 1415 & 16 & 17 & 18 & 1920 \\
\hline Ctx-Dorsal & & 0.818 & 0.867 & & & & & & & -0.627 & & & & & 0.817 & & 1 \\
\hline Ctx-Lateral & & & 0.576 & & -0.568 & & & & & & & & & & 0.842 & & 2 \\
\hline Ctx-Dorso-lateral & 0.816 & 0.804 & & -0.758 & & & & & & & & & & & & & 3 \\
\hline Medial Septal N. & & & & & & 0.871 & & & & 0.690 & & & & & & & 4 \\
\hline Globus Pallidus & & & & & & & 0.762 & & & & & & & & -0.695 & & 5 \\
\hline Lateral Septal N. & & -0.695 & -0.668 & & & & & -0.566 & & & & & & & & & 6 \\
\hline Ant. Hypothalamus & -0.675 & & & & & & & & & & & & & & -0.643 & & 7 \\
\hline Thal-Dorsomedial & & & & & & & & & 0.697 & & & & 0.567 & & & & 8 \\
\hline Thal-Vent $\mathbf{N}$. (ant med) & & & & & & & & & -0.810 & & & & & & & & 9 \\
\hline Thal-Vent N. (dorsal) & & & & & & & & & & -0.721 & & & & & & & 10 \\
\hline Ventromedial Hypothal. & & & & & & 0.733 & & & -0.663 & & & & & & -0.567 & & 11 \\
\hline Thal-Ret-Reuniens & & & & & & & & & & & & & & & & & 12 \\
\hline Thal-Mammillary & & -0.897 & -0.823 & & -0.689 & & 0.836 & & & & & & & & & & 13 \\
\hline Reticular formation & & & & & & & & & & & & & 0.755 & & & & 14 \\
\hline Substantia Nigra & & & & & & & & & & & & & & & & & 15 \\
\hline Brachium Conjunctivum & & & & & & & 0.882 & & & & & 1.00 & & & & & 16 \\
\hline N. Mes. Trigeminal Tract & & & & & & & & & & & & & & & & 0.601 & 17 \\
\hline $\begin{array}{l}\text { Deep Cerebellar } \\
\text { Nuclei }\end{array}$ & & & & -0.660 & & -0.668 & & & & & & & & & & & 18 \\
\hline Superior Colliculus & & & & & & & & & 0.756 & & & & & & & & 19 \\
\hline Caudate-Putamen & & & & & 0.757 & & & & & & & & & & & & 20 \\
\hline
\end{tabular}

Portacaval shunt animals

Correlation coefficient matrices for normal animals (top right portion) and portacaval shunt animals (bottom left portion). The first entry indicates that in the normal animals the correlation coefficient for dorsal cortex vs. dorsolateral cortex is $\mathbf{0 . 8 1 8}$. Only those correlation coefficients with $P$ values of 0.05 or less are shown. See Table $\mathrm{V}$ for results of tests for significance between correlation coefficients.

metabolism that occur after a portacaval shunt, to relate those changes to those observed after intracarotid infusions of ammonia to clarify the role of hyperammonemia in producing postshunt alterations in brain glucose metabolism, and to define potential mechanisms relevant to the development of portalsystemic or hyperammonemic encephalopathy.

Although the brain is totally dependent on glucose as its energy source under normal conditions and metabolic rates in the brain are usually reduced during periods of encephalopathy, the data concerning brain glucose metabolism and the effects of hyperammonemia are conflicting. James et al. (22) reported that whole-brain blood flow, oxygen and glucose consumption rose, then fell after portacaval shunt construction in dogs. In another publication, he reported that there was a lightening of anesthesia in normal dogs after an ammonia infusion (23). Hawkins et al. (24) reported increases in cerebral glucose consumption after the induction of acute hyperammonemia in rats. Eklof et al. (25) reported a reduction in oxygen metabolism 2 wk after portacaval a shunt in rats, and Gjedde et al. (2) found increases in flow with normal oxygen metabolism 8 wk after portacaval shunt construction. However, in these same animals there were marked reductions in cerebral blood flow and oxygen metabolism after

Table V. Probability of Difference between Normal and Portacaval Shunt Regional Correlation Coefficient

\begin{tabular}{|c|c|c|c|c|c|c|c|c|c|c|c|c|c|c|c|c|c|c|c|c|c|}
\hline Brain region & 1 & 2 & 3 & 4 & 5 & 6 & 7 & 8 & 9 & 10 & 11 & 12 & 13 & 14 & 15 & 16 & 17 & 18 & 19 & 20 & \\
\hline Ctx-Dorsal & $* * * *$ & & & & & & & & & & & & & & & & & & & & 1 \\
\hline Ctx-Lateral & & $* * * *$ & & & & & & & & & & & & & & & & & & & 2 \\
\hline Ctx-Dorso-lateral & & & $* * * *$ & & & & & & & & & & & & & & & & & & 3 \\
\hline Medial Septal N. & & & & $* * * *$ & & & & & & & & & & & & & & & & & 4 \\
\hline Globus Pallidus & & 0.006 & 0.095 & & $* * * *$ & & & & & & & & & & & & & & & & 5 \\
\hline Lateral Septal N. & & & & 0.012 & & $* * * *$ & & & & & & & & & & & & & & & 6 \\
\hline Ant. Hypothalamus & & & & & & & $* * * *$ & & & & & & & & & & & & & & 7 \\
\hline Thal-Dorsomedial & & & & & & & & $* * * *$ & & & & & & & & & & & & & 8 \\
\hline Thal-Vent N.(ant med) & & & & 0.021 & & & 0.051 & 0.012 & $* * * *$ & & & & & & & & & & & & 9 \\
\hline Thal-Vent $\mathbf{N}$ (dorsal) & & & & 0.048 & & & & & & $* * * *$ & & & & & & & & & & & 10 \\
\hline Ventromedial Hypothal. & & & & 0.026 & & & & & & & $* * * *$ & & & & & & & & & & 11 \\
\hline Thal-Ret-Reuniens & & & & & & & & & 0.001 & & & $* * * *$ & & & & & & & & & 12 \\
\hline Thal-Mammillary & & 0.052 & & 0.099 & 0.028 & & 0.081 & 0.026 & & & & & $* * * *$ & & & & & & & & 13 \\
\hline Reticular formation & & & & 0.034 & & & & & 0.014 & 0.088 & 0.052 & & & $* * * *$ & & & & & & & 14 \\
\hline Substantia Nigra & & & & & & & & & 0.030 & & & & & 0.016 & $* * * *$ & & & & & & 15 \\
\hline Brachium Conjunctivum & & & & & 0.093 & & 0.001 & & & 0.057 & & 0.040 & 0.001 & & & $* * * *$ & & & & & 16 \\
\hline N. Mes. Trigeminal Tract & 0.004 & 0.028 & & & 0.003 & & 0.013 & & 0.034 & & & 0.105 & & & & & $* * * *$ & & & & 17 \\
\hline Deep Cerebellar Nuclei & & & & & 0.100 & & & & & & & & & & & & & $* * * *$ & & & 18 \\
\hline Superior Colliculus & & & & & & & & & 0.003 & 0.033 & 0.099 & & & & & 0.001 & & & $* * * *$ & & 19 \\
\hline Caudate-Putamen & & & & & & & & & 0.031 & & 0.011 & & & & & & & & & $* * * *$ & 20 \\
\hline
\end{tabular}

Tests for significant differences between correlation coefficients. Entries in this table are the probability values associated with a test of whether the indicated metabolic correlations were different in the two groups. For example, the first entry in the second column, 0.006 , indicates that the correlation between the lateral cortex and the globus pallidus in the normal group $(r=-0.568$, see Table IV) was different from that in the portacaval shunt group $(r=0.634$, not included in table) at a level of significance equal to 0.006 . 
an ammonia load, changes not observed 4 wk after portacaval shunting, indicating an increase in the sensitivity to the toxic effects of ammonia (2). In studies of humans, Bianchi-Porro et al. (26) found increased blood flow in portacaval shunt patients who were neurologically normal, and Morgan et al. (27) reported low blood flow, glucose, and oxygen metabolic rates in encephalopathic patients with normalization after successful treatment, in substantial agreement with an earlier report by Posner and Plum (28). In more recent papers, Mans et al. (29) reported reductions in regional glucose use $8 \mathrm{wk}$ after portacaval shunting in rats whereas Cruz and Duffy (8) reported increases in glucose under experimental conditions that appear to be identical, with the exception of the method used to measure glucose use.

We measured the glucose metabolic rate in 20 brain regions and found significant increases in each region $8 \mathrm{wk}$ after portacaval shunt construction. The increases were smallest in the cortex, ranging from 12 to $18 \%$ above the control value, were larger in the thalamic, hypothalamic and basal ganglionic regions, and were greatest in the reticular formation where glucose use was increased to $74 \%$ above the control value. These findings are in substantial agreement with those of Cruz and Duffy (8). 8 wk after portacaval shunt construction they reported glucose use was increased in all but five of the 36 structures they surveyed. These increases reached statistical significance in 13 brain regions that in general were similar to the regions in which we observed maximal effects. We did not observe decreases in the glucose metabolic rates in portacaval animals as reported by Mans et al. (29), or in animals (both normal and portacaval shunt) subjected to threshold ammonia infusions via the carotid artery.

Effects of ammonia on brain glucose metabolism. Since we desired to examine threshold metabolic events attributable to ammonia infusions, the amount of ammonia that was infused was not massive, as was the case in the studies of Hawkins et al. (24), where brain ammonia levels increased from 0.2 to 1.53 $\mathrm{mM}$. As might be expected, the results of our glucose metabolism studies varied: not all animals showed a metabolic response to the infusion and the intensity of the response varied. These observations are consistent with the frequency with which electroencephalographic changes were seen, the variability in the ${ }^{13} \mathrm{~N}$-ammonia infusion studies, which we believe to cause the variability of the ammonia response, and observations of unanesthetized, ammonia-infused animals. Visual inspection of the quantitative images of cerebral glucose consumption indicated that about half of the normal and half of the animals with portacaval shunts exhibited increases in glucose use in the infused hemisphere: unilateral decreases in the glucose metabolic rate were not observed. We conclude that in normal and portacaval shunt animals, brain glucose metabolism is increased by relatively small increments in the amount of ammonia delivered to the brain when infusion schedules are designed to avoid possible behavioral effects of ammonia. Individual brain regions vary in their susceptibility to this effect. Adding $0.05 \mu \mathrm{M}$ of ammonia per gram of brain per minute appears to be the threshold for observing a metabolic effect. The occasionally dramatic nature of ammonia effect is shown in Fig. 1, where the glucose metabolic rate in the substantia nigra of the infused hemisphere was more than three times greater than in the contralateral nigra. It seems likely that more severe hyperammonemia, especially in the portacaval shunt animals, would produce coma, as observed in earlier studies (2), and that under these more extreme conditions glucose metabolism would be reduced.

In addition to hyperammonemia, portacaval shunts produce a variety of metabolic changes in both rats and humans, and many of these changes have been suggested as factors that produce encephalopathy. Alterations in the plasma branched chain/ aromatic amino acid ratio (30) and increases in gamma-amino butyric acid occur (31) and have been held to be etiologically responsible for the production of encephalopathy. We were reluctant to attribute the increases in brain glucose metabolism observed in the portacaval animals to hyperammonemia on the basis of a correlation analysis relating brain glucose metabolism in the two groups to the blood ammonia concentration, since any two variables that occur with predictable regularity after a shunt would almost certainly correlate at a statistically significant level, regardless of whether a cause-and-effect relationship existed. However, we believe that the results of the intracarotid infusion studies provide convincing evidence to link the observed changes in brain glucose metabolism with hyperammonemia. Not only did small amounts of exogenous ammonia increase brain glucose metabolism, but the increase occurred in a pattern that was very similar to the metabolic pattern observed after a portacaval shunt.

Since enzymes related to ammonia metabolism and neuropathological changes after hyperammonemia are confined to astrocytes, Norenberg (6) has argued that the nervous system dysfunction after hyperammonemia can be attributed to astrocytic abnormalities. The nuclear specificity observed in the present studies suggests that there may be a neuronal component as well. Since the metabolic response to hyperammonemia and portacaval shunting is not generalized but is specific to nuclei or nuclear groups, it is possible that brain regions differing in their cytoarchitectural characteristics differ in their response to exogenous ammonia. The actions of ammonia on the brain are complex: in addition to metabolic abnormalities already cited, the response includes changes in membrane electrical properties, thought to be attributable to chloride pump inhibition (32). Interactions between metabolic and physiological abnormalities in mixed glial and neuronal populations that use different neurotransmitters, such as those in the substantia nigra, may explain the nuclear specificity to exogenous ammonia.

Implications for the pathogenesis of hyperammonemic encephalopathy. The differences in regional susceptibility to the effects of ammonia implied by our data analysis suggest that portacaval shunting and hyperammonemia affect the metabolism, and presumably the function, of individual cerebral nuclei and fundamental patterns of cerebral interaction. These effects may be of importance in the development of the clinical signs and symptoms of hepatic encephalopathy.

The hypothesis that there are differences in regional cerebral interactions of normal vs. portacaval shunt animals is strengthened by an inspection of the results of the principal component analysis shown in Table III, the tabulated correlation coefficients shown in Table IV, and the test for differences between correlation coefficients shown in Table $\mathrm{V}$. The extracted factors (A, and $B$ in Table III) describe major dimensions along which the measured regional metabolic rates covary. The congruence coefficients show that the factor loading values shown in the table are significantly dissimilar. Beyond this, objective criteria are not available to describe the results of this analysis, but inspection of the coefficients (magnitude and sign) and determining which of the two factors predominates for a given brain region (greatest absolute magnitude) provides insight into the degree of similarity between the post-shunt and normal metabolic relationships. Data from populations with identical interactions would yield identical 
coefficients for both extracted components. Inspection of the correlation coefficients from the principal component analysis reveals very few similarities, which suggests that there are fundamental differences in metabolic interactions after a portacaval shunt, as implied by the computed congruence coefficients. Similarly, the large number of significant differences in correlation coefficients shown in Table $\mathrm{V}$ supports our hypothesis that there is a difference between the "metabolic anatomy" of normal vs. portacaval shunted rats. The protean nature of the signs and symptoms of hepatic encephalopathy in humans, including altered levels of consciousness, impaired cognition, movement disorders, corticospinal tract dysfunction with a Babinski response, and occasional epileptic seizures may be the result of alterations in functional cerebral interactions implied by the analysis of glucose metabolic patterns.

The largest increase in the glucose metabolic rate after a portacaval shunt was observed in the reticular formation, the portion of the brain responsible for maintaining consciousness. The reticular formation has been identified by McCandless and Schenker (5) as being highly sensitive to ammonia, since ATP, phosphocreatine, and glucose levels were selectively decreased in the reticular formation of mice during the precoma period of acute ammonia intoxication (5). Our data showing rapid glucose use by the reticular formation after a portacaval shunt are consistent with their concept of selective vulnerability of the reticular formation to ammonia, and imply a link between the effects of ammonia on the reticular formation and the pathogenesis of coma.

Weight loss and cachexia are commonly observed after a portacaval shunt in animals $(2,8,12)$ and in terminal liver disease in humans. The link between the hypothalamus, feeding behavior, and weight gain or loss was established in classical studies reported by Brobeck and his associates (33). Morgane expanded these studies, described a series of feeding behaviors that depend on the activity of neural elements in and around the hypothalamus and medial forebrain bundle, and suggested that hunger drive originating in the mediolateral hypothalamus is suppressed by stimulating ventromedial hypothalamic centers (34). It is possible that the ammonia-induced neural activation we observed in the ventromedial hypothalamus (see Figs. 1 and 2) inhibits hunger mechanisms and leads to weight loss. An additional more direct link between hyperammonemia and feeding behavior was reported by Noda and Chikamori who observed reductions in food intake after ammonium chloride injections into the prepyriform cortex (35). Thus, hyperammonemia may lead to reductions in food intake by direct or indirect actions on cerebral satiety control centers. Weight loss and cachexia would follow. Lockwood et al. (1) found that substantial amounts of ammonia are metabolized and detoxified by skeletal muscle. In patients with end-to-side portacaval shunts, skeletal muscle was the primary organ for ammonia detoxification (1). Therefore, loss of muscle bulk could then make an individual more likely to develop hyperammonemia. This, combined with increasing ammonia sensitivity, may lead to the development of a selfperpetuating cycle of hyperammonemia, appetite suppression, loss of additional skeketal muscle bulk, a further reduction in the ammonia detoxification capacity, and hyperammonemia.

Portal systemic encephalopathy is a syndrome with multiple neurological manifestations. We believe that the results of the present study strengthen the hypothesis that ammonia is of great importance in the development of this disorder via direct actions on specific nuclei, such as the ventromedial hypothalamus or the ascending reticular activating system, coupled with ammonia induced alterations in normal functional interactions in the brain as reflected by changes in the pattern of cerebral glucose metabolism.

\section{Acknowledgments}

We are grateful to David Smith who wrote computer programs for image analysis of autoradiograms and to Dr. John Overall for his valuable advice concerning the statistical presentation of the data.

This study was supported in part by Positron Tomography Center grant NS 15639, Cerebral Vascular Disease Research Center grant NS 05820, National Institutes of Health grant AM 38307, and the Cullen Trust for Health Care, Houston, TX.

\section{References}

1. Lockwood, A. H., J. M. McDonald, R. E. Reiman, A. S. Gelbard, J. S. Laughlin, T. E. Duffy, and F. Plum. 1979. The dynamics of ammonia metabolism in man: effects of liver disease and hyperammonemia. $J$. Clin. Invest. 63:449-460.

2. Gjedde, A., A. H. Lockwood, T. E. Duffy, and F. Plum. 1978. Cerebral blood flow and metabolism in chronically hyperammonemic rats: effect of an acute ammonia challenge. Ann. Neurol. 3:325-330.

3. Hindfelt, B., F. Plum, and T. E. Duffy. 1977. Effect of acute ammonia intoxication on cerebral metabolism in rats with portacaval shunts. J. Clin. Invest. 59:386-396.

4. Lockwood, A. H. 1985. Ammonia-induced encephalopathy. In Cerebral Energy Metabolism and Metabolic Encephalopathy. D. W. McCandless, editor. Plenum Press, NY. 203-227.

5. McCandless, D. W., and S. Schenker. 1980. Effect of acute ammonia intoxication on energy stores in the cerebral reticular activating system. Exp. Brain Res. 44:325-330.

6. Norenberg, M. D. 1984. The astrocyte in liver diseases. Adv. Cell Neurobiol. 2:303-351.

7. Walker, C. O., and Schenker, S. 1970. Pathogenesis of hepatic encephalopathy with special reference to the role of ammonia. Am. J. Clin. Nutr. 23:619-632.

8. Cruz, N. F., and T. E. Duffy. 1983. Local cerebral glucose metabolism in rats with portacaval shunts. J. Cereb. Blood Flow Metabol. 3: 311-320.

9. Lockwood, A. H., R. D. Finn, J. A. Campbell, and T. B. Richman. 1980. Factors that affect the uptake of ammonia by the brain: the bloodbrain pH gradient. Brain Res. 181:259-266.

10. Lockwood, A. H., L. Bolomey, and F. Napoleon. 1984. The blood-brain barrier to ammonia in humans. J. Cereb. Blood Flow Metabol. 4:516-522.

11. Lockwood, A. H., M. D. Ginsberg, C. M. Butler, and M. T. Gutierrez. 1982. Selective effects of ammonia on regional brain glucose metabolism. Ann. Neurol. 12:144.

12. Lee, S. H., and B. Fisher. 1961. Portacaval shunt surgery in the rat. Surgery. 50:668-672.

13. Folbergrova, J., J. V. Passonneau, O. H. Lowry, and D. W. Schulz. 1969. Glycogen, ammonia and related metabolites in the brain during seizures evoked by methionine sulfoximine. J. Neurochem. 16:191-203.

14. Sokoloff, L., M. Reivich, C. Kennedy, M. H. Des Rosiers, C. S. Patlak, K. D. Pettigrew, O. Sakurada, and M. Shinohara. 1977. The ${ }^{14} \mathrm{C}$ deoxyglucose method for the measurement of local cerebral glucose utilization: theory, procedure, and normal values in the conscious and anesthetized albino rat. J. Neurochem. 28:897-916.

15. Savaki, H. E., L. Davidsen, C. Smith, and L. Sokoloff. 1980. Measurement of free glucose turnover in brain. J. Neurochem. 35:495502.

16. Goochee, C., W. Rasband, and L. Sokoloff. 1980. Computerized densitometry and color coding of ${ }^{14} \mathrm{C}$ deoxyglucose autoradiographs. Ann. Neurol. 7:359-370.

17. Pellegrino, L. J., A. S. Pellegrino, and A. J. Cushman. 1979. A Stereotoxic Atlas of the Rat Brain, 2nd Ed. Plenum Press, NY. 
18. Overall, J. E., and C. J. Klet. 1972. Applied Multivariate Analysis. McGraw-Hill, Inc., New York. 111-126.

19. Cattel, R. B. 1978. The Scientific Use of Factor Analyses. Plenum Press, Inc., New York. 229-270.

20. Bruning, J. L., and B. L. Kintz. 1968. Computational Handbook of Statistics. Scott, Foresman and Co., Glenview, IL. 191.

21. Centers for Disease Control. 1983. Annual Summary 1982: reported morbidity and mortality in the United States. Morbidity and Mortality Weekly Rep. 31(54):110-111.

22. James, I. M., C. MacDonell, and C. Xanalatos. 1972. The effect of acute portacaval shunting in dogs on cerebral and peripheral blood flow and metabolism. Clin. Sci. 42:769-774.

23. James, I. M., C. MacDonell, and C. Xanalatos. 1974. Effects of ammonium salts on brain metabolism. J. Neurol. Neurosurg. Psychiat. 37:948-953.

24. Hawkins, R. A., A. L. Miller, R. C. Nielsen, and R. L. Veech. 1973. The acute action of ammonia on rat brain in vivo. Biochem. $J$. 134:1001-1008.

25. Eklof, B., T. Holmin, H. Johannsson, and B. K. Siesjo. 1974. Cerebral blood flow and cerebral metabolic rate for oxygen in rats with portacaval anastomosis. Acta. Physiol. Scand. 90:337-344.

26. Bianchi-Porro, G., A. T. Maiolo, and P. Della Porto. 1969. Cerebral blood flow and metabolism in hepatic cirrhosis before and after portacaval shunt operation. Gut. 10:894-897.
27. Morgan, M., A. W. Jakobovits, I. M. James, and S. Sherlock. 1980. Successful use of bromocriptine in the treatment of chronic hepatic encephalopathy. Gastroenterology. 78:663-670.

28. Posner, J. B., and F. Plum. 1960. The toxic effects of carbon dioxide and acetazolamide in hepatic encephalopathy. J. Clin. Invest. 39:1246-1258.

29. Mans, A. M., J. F. Biebuyck, D. W. Davis, R. M. Bryan, and R. A. Hawkins. 1983. Regional cerebral glucose utilization in rats with portacaval anastomosis. J. Neurochem. 40:986-991.

30. Fischer, J. E., H. M. Rosen, A. M. Ebeid, J. H. James, J. M. Keane, and P. B. Soeters. 1976. The effect of normalization of plasma amino acids on hepatic encephalopathy in man. Surgery. 80:77-91.

31. Jones, A. E., D. F. Schafer, P. Ferenci, and S. C. Pappas. 1904. The neurobiology of hepatic encephalopathy. Hepatology. 4:1235-1242.

32. Lux, H. D. 1971. Ammonium and chloride extrusion: hyperpolarizing synaptic inhibition in spinal cord motoneurons. Science (Wash. DC) 173:555-557.

33. Anand, B. K., and J. R. Brobeck. 1951. Hypothalamic control of food intake in rats and cats. Yale J. Biol. Med. 24:123-140.

34. Morgane, P. J. 1961. Electrophysiological studies of feeding and satiety enters in the rat. Am. J. Physiol. 201:838-844.

35. Noda, K., and K. Chikamori. 1976. Effect of ammonia via prepyriform cortex on regulation of food intake in the rat. Am. J. Physiol. 231:1263-1266. 